\title{
Trans-National Joint Research Projects
}

\section{Defying the Odds of National Inter-University Competition}

\author{
Armin Heinzl • Martin Bichler • \\ Wil M. P. van der Aalst
}

Published online: 17 May 2017

(C) Springer Fachmedien Wiesbaden 2017

The past is always different than the future. Three decades ago, disciplinary communities were focused on their home country. "Wirtschaftsinformatik", the representative of the German speaking Information Systems community, was eager to present itself to the chorus of the established disciplines as a novel but ambitious field of research. As a consequence, the number of university programs and positions in Business and Information Systems Engineering was not merely growing but its protagonists were eager to conduct prestigious and valuable large scale research projects funded by renowned institutions. Some of our readers may well remember the following projects (see also Heinrich et al. 2011, p. 192):

- Interactive Business Information and Control Systems, focus program of the German Science Foundation, 1984-1990,

- Distributed Information Systems in Business, focus program of the German Science Foundation, 1991-1997,

Prof. Dr. A. Heinzl ( $\square)$

Chair of General Management and Information Systems,

University of Mannheim, 68161 Mannheim, Germany

e-mail: heinzl@uni-mannheim.de

Prof. Dr. M. Bichler

Department of Informatics, Decision Sciences and Systems, Technical University of Munich (TUM), Boltzmannstr 3, 85748 Munich, Germany

e-mail: bichler@in.tum.de

Prof. dr. ir. W. M. P. van der Aalst

Department of Mathematics and Computer Science (MF 7.103),

Eindhoven University of Technology, PO Box 513,

5600 MB Eindhoven, The Netherlands

e-mail: w.m.p.v.d.aalst@tue.nl
- Intelligent Agents and their Application Scenarios in Business, focus program of the German Science Foundation, 2000-2006, or

- Internet Economics, focus program of German Ministry of Education and Research, 2003-2007.

All of these exemplary projects comprised up to two dozen of principal investigators and required a significant amount of conceptual preparation and thought leadership. This led to the fact that these intra-disciplinary but cross-university consortia were able to compete with project proposals from traditional disciplines and succeed in highly demanding evaluation and selection processes. As a consequence, these large scale projects were able to win recognition from all national scientific communities and developed a substantial level of disciplinary coherence and pride among the participating investigators. Furthermore, they offered young talented researchers a rich environment to become senior scientists.

However, since 2007, large scale research projects in Information Systems have almost vanished from the scene. What are the key drivers of this evolutionary change? According to our perceptions, there are several factors which may have caused this inversion:

1. The "publication game" has gained more salience by overriding the efforts for conceptualizing and conducting new large scale projects. The focus is on generating publications in specific journals rather than building larger consortia across disciplines.

2. Younger researchers are stimulated to distinguish themselves from their environment. To obtain tenure, researchers need to show that they are able to acquire funding as an individual.

3. Inter-university competition, stimulated by global education markets and government programs like the German Excellence Initiative, urge researchers to be 
more loyal to their employing institutions rather than engaging into disciplinary but location-spanning projects. Interestingly, the first phase of the Excellence Initiative started in 2007.

4. Research on Business and Information Systems Engineering is considered to be of high practical relevance. This is good, but also creates a climate where companies are expected to fund research (unlike astronomy, physics, etc.). As a result, there is less room for large government funded initiatives. Industry funded PhDs tend to work on mini-projects tailored towards a specific company.

5. The Information Systems community has further developed towards theme- and method-specific sub-communities that have become partially incommensurable. The sub-community $\mathrm{A}$ has difficulties in finding a common denominator with sub-community $\mathrm{B}$ and $\mathrm{C}$ in order to gain momentum for a novel significant research project.

It is hard to tell which of these factors have been most prominent. Probably, it is the combination of forces that created the current vacuum in large scale research projects within our community. In the meantime, scientific disciplines have become more international. In particular, the European Union provides significant frameworks for the funding of trans-national research initiatives. Nevertheless, the factors listed above fail also to nourish our discipline from a European point of view.

For instance, a search on the EU database CORDIS ${ }^{1}$ for "Information System" revealed 33 interesting projects within the ongoing Horizon 2020 program. 14 of these projects documented investigators from Germany and eight projects listed investigators from the Netherlands. A closer look at the program descriptions reveals a rather disillusioning outcome. The projects deal - among others - with electronics, health topics, genetic resources, earth observation data, food security, deep-sea ecosystems, and IT security.

Another search for individual projects on "Information Systems" funded by the European Research Commission ${ }^{2}$ revealed a similar picture. Among the 89 ERC funded projects, there are virtually none which refer to our disciplinary community. Unfortunately, it seems that the visibility of the entire field has room for improvement. To evaluate ERC grants, there are panels for management, marketing, organizational behavior, and operations management, but there is no panel for Information Systems. This corresponds to the observation that some of the top international research universities including Berkeley, Harvard, Stanford, Yale, or Princeton do not maintain IS groups. This provides further motivation to work on joint projects that strengthen the identity and visibility of our field.

Large scale disciplinary projects offer substantial working environments, yield a critical mass of input resources for developing high quality research output which is publishable in top international and national publication outlets. Thus, we believe that future efforts in re-accessing this domain are essential - for the reputation of individual researchers and the reputation of our community. Both elements - the individual and the community - mutually reinforce each other. Outstanding researchers advance the community - and a successful community attracts and nurtures bright young talents which in turn boost the quality level of the entire collective including the outstanding researchers.

Thus, BISE highly welcomes a re-intensification of such efforts on a trans-national level and offers committed researchers opportunities for exposure. Investigators who are willing to take such efforts to the next level may publish facets of their initiatives in the discussion, catchword, or interview section before the first results materialize. At a later point of time, of course, they are invited to submit their research results to one of the BISE departments for demonstrating the substance of such efforts. May the future be more promising than the past!

\section{Reference}

Heinrich LJ, Heinzl A, Riedl R (2011) Wirtschaftsinformatik: Einführung und Grundlegung. Springer, Heidelberg

${ }^{1}$ http://cordis.europa.eu/home_en.html.

${ }^{2}$ https://erc.europa.eu/projects-figures/erc-funded-projects. 